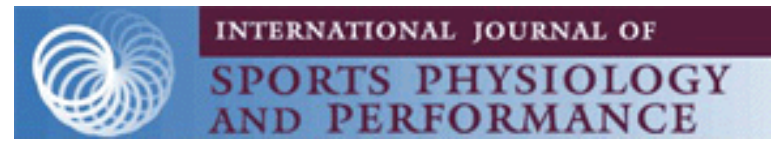

\title{
Measurement characteristics of athlete monitoring tools in professional Australian football
}

\begin{tabular}{|r|l|}
\hline Journal: & International Journal of Sports Physiology and Performance \\
\hline Manuscript ID & IJSPP.2019-0060 \\
\hline Manuscript Type: & Original Investigation \\
\hline Author: & $22-J a n-2019$ \\
\hline Complete List of Authors: & $\begin{array}{l}\text { Ryan, Samuel; University of Technology Sydney, Human Performance } \\
\text { Research Centre; Carlton Football Club } \\
\text { Pacecca, Emidio; Carlton Football Club } \\
\text { Tebble, Jye; Carlton Football Club } \\
\text { Hocking, Joel; Carlton Football Club } \\
\text { Kempton, Tom; Carlton Football Club } \\
\text { Coutts, Aaron; University of Technology Sydney, Human Performance } \\
\text { Research Centre; Carlton Football Club }\end{array}$ \\
\hline Keywords: & \begin{tabular}{l} 
monitoring, reliability, sensitivity \\
\hline
\end{tabular} \\
\hline
\end{tabular}

\section{SCHOLARONE ${ }^{\text {M }}$ Manuscripts}


Manuscript title: Measurement characteristics of athlete monitoring tools in professional Australian football

Submission type: Original investigation

Authors: Samuel Ryan ${ }^{\mathrm{ab}}$, Emidio Pacecca ${ }^{\mathrm{b}}$, Jye Tebble $^{\mathrm{b}}$, Joel Hocking ${ }^{\mathrm{b}}$, Thomas Kempton ${ }^{\mathrm{b}}$ and Aaron J. Coutts ${ }^{\mathrm{ab}}$

\title{
Affiliations:
}

a University of Technology Sydney (UTS), Human Performance Research Centre, Sydney, AUSTRALIA

${ }^{\mathrm{b}}$ Carlton Football Club, Melbourne, AUSTRALIA

Corresponding Author: Samuel Ryan Ph: 0417484884

e-mail:sam.ryan@carltonfc.com.au

\begin{abstract}
word count: $\quad 241$
\end{abstract}
Word count: $\quad 3347$

Number of tables: $\quad 3$

Number of figures: $\quad 0$ 


\section{Abstract}

2

3 Purpose: To examine the measurement reliability and sensitivity of common athlete monitoring tools in professional AF players.

5

6 Methods: Test-retest reliability (noise) and weekly variation (signal) data were collected from

742 professional Australian footballers from one club during a competition season. Perceptual 8 wellness was measured via questionnaires completed before main training sessions (48, 72 and

$996 \mathrm{~h}$ post-match), with players providing a rating (1-5 Likert scale) regarding their muscle 10 soreness, sleep quality, fatigue level, stress and motivation. Eccentric hamstring strength and 11 countermovement jump performance was assessed via proprietary systems once per week. 12 Heart rate recovery (HRR) was assessed via a standard submaximal run test on a grass-covered 13 field with players wearing a heart rate monitor. The HRR was calculated by subtracting average 14 heart rate during final $30 \mathrm{~s}$ of exercise from average heart rate during final $10 \mathrm{~s}$ of rest. Typical 15 test error was reported as coefficient of variation (CV\% and TE). Sensitivity was calculated by 16 dividing weekly $\mathrm{CV}$ by test $\mathrm{CV}$ to produce a signal-to-noise ratio (SNR).

18 Results: All measures displayed acceptable sensitivity. SNRs ranged from 1.3-11.1. ICCs ranged from 0.30 to 0.97 for all measures.

Conclusions: The HRR test, CMJ test, eccentric hamstring strength test and perceptual wellness all possess acceptable measurement sensitivity. SNR analysis is a novel method of assessing measurement characteristics of monitoring tools for professional AF players. These data can be used by coaches and scientists to identify meaningful changes in common measures of fitness and fatigue.

Key Words: athlete monitoring, reliability, sensitivity 


\section{Introduction}

29 Athlete monitoring systems are commonly used in professional team sports to provide coaches and scientists with an understanding of player performance readiness and injury risk. ${ }^{1-3}$ Information from these systems is used to plan training load to maximise adaptations whilst maintaining player availability for competition. ${ }^{4}$ Recent research and technological advances has increased the number of tools available to assess constructs of fitness and fatigue. These include submaximal heart rate tests, ${ }^{5}$ countermovement jump tests, ${ }^{6}$ lower limb muscular strength tests ${ }^{7}$ and perceptual wellness questionnaires. ${ }^{8}$

Due to environmental constraints and risk of injury in fatigued athletes, it is impractical for professional team sport athletes to complete maximal physical capacity tests during the season to determine changes in fitness and fatigue. ${ }^{9}$ Therefore, practitioners rely on monitoring tools that are submaximal in nature and can identify changes in constructs of fitness and fatigue regularly throughout training and competition to assess the performance readiness of their players. To provide useful information to coaches and scientists, these tools should display measurement characteristics of validity (the ability of a test to measure what it is designed to measure), reliability (the consistency of results from a test) and sensitivity (the extent to which a test can detect changes beyond the typical error in results). ${ }^{10,11}$ Reliability can be assessed via test-retest analysis, where measurements are collected from the same individuals under

47 identical test conditions. ${ }^{12}$ This produces a typical error measure, often expressed as a coefficient of variation percentage $(\mathrm{CV} \%)$ that indicates the level of error to be accounted for when interpreting changes in that test. ${ }^{13}$ Using the $\mathrm{CV} \%$, the sensitivity of a test can be established via signal-to-noise anlysis. ${ }^{12}$ Indeed, measurement signal is often assessed via intervention studies where responsiveness (i.e. a change in performance) is measured following the intervention, ${ }^{14}$ however this is not possible in professional team sport environments due to 
time and cost constraints. Therefore, in the case of team sport athlete testing, "signal" refers to individual changes in a monitoring test in response to training stimuli (provided by a valid test), while "noise" is represented by the typical error in the measurement (from test-retest reliability analysis). Measurement signal and noise can be combined to produce a signal-to-noise ratio (SNR), providing practitioners with an index of responsiveness in a measure relative to the typical error in the test. This information is important to coaches and scientists as it allows confident interpretation of athlete monitoring data by identifying meaningful changes (i.e. those that exceed the "noise" in the test).

Studies in professional Australian Football (AF) have shown perceptual wellness questionnaires are sensitive to weekly change in training load ${ }^{8}$ and match load, ${ }^{15}$ suggesting they are valid measures of training response in this athletic population. However, the measurement characteristics of perceptual wellness questionnaires has received little research attention. A study of collegiate basketballers reported a total wellness test CV\% of $6.9,{ }^{6}$ while research in professional AF reported a Chronbach's alpha of 0.87 as a measure of reliability on a composite scale of nine wellness constructs. ${ }^{8}$ These findings indicate that perceived wellness questionnaires possess acceptable reliability as measured by $\mathrm{CV} \%,{ }^{12}$ however the capability of these questionnaires to detect changes that exceed the typical error is unknown. Moreover, no research has examined the reliability of perceived wellness using individualised z-scores. Additionally, while the Nordic eccentric hamstring strength test possesses acceptable reliability as a measure of hamstring force production and can discriminate between previously injured and uninjured athletes, ${ }^{16}$ the sensitivity of this test in professional AF players has not been established. 
77 Submaximal heart rate tests may be administered at regular intervals to provide practitioners

with a non-fatiguing assessment of changes in aerobic fitness in team sport athletes. ${ }^{17}$ Previous research in professional AF reported a submaximal heart rate recovery test to be a valid and reliable measure of training status. ${ }^{17}$ However, the capacity of this test to detect changes exceeding the typical error has not been examined in this athletic population. Additionally, countermovement jump tests (CMJ) are commonly used to assess neuromuscular fatigue in professional AF players. ${ }^{18,19} \mathrm{CMJ}$ performance has been shown to be responsive to match load, with substantial reductions in CMJ flight time following competition matches, ${ }^{19}$ while another study reported decreases in $\mathrm{CMJ}$ performance were related to increases in low-speed movement and reduced accelerations during competition matches. ${ }^{18}$ Moreover, CMJ performance has been shown to demonstrate acceptable measurement reliability in team sport athletes, with $\mathrm{CV}$ ranging from $1.1 \%$ to $7.1 \%$ across a range of jump variables..$^{20}$ However, no research has investigated if weekly variation in CMJ performance exceeds the typical error in this test among professional AF players.

Collectively, research suggests that perceptual wellness questionnaires, eccentric hamstring strength tests, CMJ tests and submaximal heart rate tests possess varying levels of reliability among a range of athlete cohorts. However, the extent to which changes in these tests exceed their typical error remains unknown, limiting interpretability of test results for coaches and scientists. Therefore, the purpose of this study was to establish the reliability and sensitivity of common monitoring tests in a professional AF population. This information will allow coaches and scientists of professional AF teams to confidently identify and interpret meaningful changes in commonly collected monitoring data. 
Methods

103 Data were collected from 45 professional Australian footballers (age: $24.6 \pm 4.0 \mathrm{y}$; height: 1.88

$104 \pm 0.07 \mathrm{~m}$; weight: $86.0 \pm 9.0 \mathrm{~kg}$ ) from one club during the 2018 AFL competition season (week

105 prior to round 1 to round 23, i.e. March to August). Informed consent and institutional ethics

106 approval were obtained (UTS HREC: ETH17-1942). Reliability and weekly variation testing

107 protocols were identical for all four tests. The number of subjects varied between measurement

108 tests and is reported in Table 2 and Table 3.

111 Players completed a short questionnaire on a smartphone device before the main field training session (7:00 to 9:00) prior to each competition match, prompting them to provide a rating

113 from 1 to 5 ( 1 representing a low or poor rating and 5 representing a high or good rating) in

114 relation to their perception of muscle soreness, sleep quality, fatigue level, stress and

115 motivation. The questionnaire used in this study was customised for the observation group

116 based on a common protocol used in previous research. ${ }^{21}$ Test-retest reliability was conducted

117 using an identical protocol on a main training day ( $96 \mathrm{~h}$ post-match in the final week of

118 competition), approximately 30 minutes after their initial completion of the questionnaire,

119 consciously avoiding recall of their previous responses. This method of reliability assessment

120 was based on previous research in elite athletes. ${ }^{22}$ All perceptual wellness responses were

121 reported relative to players' individual mean and standard deviation as a z-score. ${ }^{15}$

123 Eccentric hamstring strength 
124 Eccentric hamstring strength was assessed once per week (72 hours post-match) in the afternoon following the main skills training session of the week using a proprietary hamstring strength testing system (Nordbord, Vald Performance, Albion, Australia). Players placed their

127 feet inside two hooks at the back of the Nordbord (superior to the lateral malleolus of each ankle) and slowly moved their torso forward, with their bodyweight eliciting contraction of the hamstring muscle group. Once in a near-flat prone position, players placed their hands in front of themselves and gently fell toward the floor. Verbal cues were provided to prompt a $50 \%$ warm up repetition (i.e. not maximal effort), followed by three maximum effort repetitions.

132 Test results were analysed by limb force in Newtons (left, right and average). This protocol 133 was based on a previous study using a customised apparatus, ${ }^{16}$ however no research has established specific protocols for the system used in this study. Test-retest reliability was conducted using an identical protocol in the afternoon of a typical training day, with maximal tests separated by three minutes of static recovery. ${ }^{16}$

\section{CMJ performance}

139 CMJ performance was assessed once per week (96 hours post-match) during a strength training session in the afternoon following the main skills session of the week during the competition season. Players held a wooden rod $(12 \times 1200 \mathrm{~mm})$ across their shoulders and were instructed to choose a depth where they felt they could jump as high as possible. Verbal cues were

143 provided to prompt a 50\% warm up repetition, followed by three maximum effort repetitions

144 from the same starting position. This protocol was based on previous research using similar testing systems. ${ }^{23,24}$ Force and jump height were measured by a proprietary force plate system

146 (ForceDecks, Vald Performance, Albion, Australia) with a sampling rate of $1000 \mathrm{~Hz}$. Test-

147 retest reliability was conducted using an identical protocol in the afternoon of a main training

148 day, with tests separated by five minutes of passive recovery. ${ }^{23}$ Variables chosen for analysis 
were based on previous studies in professional AF players ${ }^{20}$ and collegiate athletes, ${ }^{23}$ including peak jump height, mean concentric force, reactive strength index, relative peak power and relative peak force.

\section{Submaximal heart rate test}

154 Submaximal heart rate tests were conducted on eight occasions throughout the competition season (48 hours post-match) as part of a warm up for the main skills session of the week. Players were instructed to run back and forth on a grass-covered field over 80-m intervals for

157 five minutes at a submaximal speed $\left(12 \mathrm{~km} \cdot \mathrm{h}^{-1}\right)$ while wearing a heart rate monitor (Polar T31

158 Wireless Heart Rate Monitor, Polar Australia). Players were prompted by a beep at the end of 159 each running interval to ensure they maintained the correct running speed. Following the exercise protocol, players were instructed to sit on the ground and remain still for $60 \mathrm{~s}$. Heart rate data was captured using $10 \mathrm{~Hz}$ Global Positioning System (GPS) units worn by each individual player between their scapulae within a small pouch in their training jersey, and

163 downloaded using proprietary software (Openfield 1.20.0, Catapult Sports, Melbourne, 164 Australia) following each test. The average heart rate (beats per minute) of each player in the final 30 seconds of the test period (HRex) and in the final $10 \mathrm{~s}$ of the $60 \mathrm{~s}$ recovery period (HRR) were collected. ${ }^{17}$ Heart rate recovery was calculated by subtracting the average heart rate (beats per minute) during the final $30 \mathrm{~s}$ of the heart rate test from the average heart rate during the final $10 \mathrm{~s}$ of the $60 \mathrm{~s}$ rest period. Individual maximum heart rate values were derived

169 from maximal testing (2-km time trial) conducted during the preseason training period. ${ }^{25}$ Testretest reliability was conducted using an identical protocol, with tests separated by a nontraining day (48 hours) during the preseason training period. 
174 Data were exported from proprietary software and collated in a customised Microsoft Excel spreadsheet (Microsoft, Redmond, USA). Test-retest reliability was assessed using customised spreadsheets ${ }^{26}$ to calculate the typical error, expressed as a coefficient of variation percentage

177 (CV\%), and intraclass coefficient (ICC) for CMJ test, eccentric hamstring test and HRR test.

178 Perceived wellness reliability was calculated using the same spreadsheets to generate a TE

179 (typical error) value, as z-scores did not require log-transformation. Normality of wellness data was confirmed via inspection of histograms. Typical weekly variation was assessed using the same custom spreadsheets (CV\%). ${ }^{26}$ Weekly perceptual wellness was categorised by number of hours post-match (48, 72 and 96 hours) as it was the only measure collected at multiple time points within a training week. The SNRs for the four tests at each time point were calculated by dividing weekly variation by the CV or TE established via test-retest reliability. Mean, standard deviation and 90\% confidence intervals were also calculated. ${ }^{12}$ SNRs were assessed as "poor" if $<1.0$, "acceptable" if 1.0-1.5, and "good" if $>1.5$, adapted from research in other professional sports. $^{22,27}$

\section{Results}

Test-retest reliability results for perceptual wellness, eccentric hamstring strength, CMJ and heart rate recovery are shown in Table 1 . Weekly variation and SNRs are shown in Tables 2 and 3. All monitoring measures at all time points displayed acceptable to good SNRs. ICCs ranged from 0.30 to 0.97 across all other measures.

\section{Discussion}


200 The aim of this study was to establish the measurement reliability and sensitivity of common monitoring tests in professional AF players. Our findings show that the heart rate recovery test,

202 CMJ test, eccentric hamstring strength test and perceptual wellness test all possess acceptable sensitivity and therefore can confidently be used by coaches and scientists of professional AF teams to identify meaningful changes in constructs of fitness and fatigue in their players.

The present results showed that all wellness measures displayed acceptable SNRs at 48, 72 and 96 hours post-match, with perceived stress displaying the greatest sensitivity (SNR: 1.3 to 11.1). Notably, perceived stress and perceived soreness were the only two elements to display SNRs of $>2.0$ at any time point, suggesting that these are the most responsive to training stressors of the five wellness elements examined in this study. Interestingly, perceived stress displayed the equal-lowest SNR at 96 hours post-match, suggesting that factors affecting player stress levels were most influential at 48 and 72 hours post-match, possibly related to the

213 previous week's match. Overall, SNRs for all wellness elements were lower (i.e. a weaker signal) at 96 hours post-match than at earlier time-points, with perceived soreness the only element to display a SNR of $>1.5$, indicating that players had stable perceptions of stress, motivation, sleep quality and fatigue within 96 hours post-match. This is in agreement with

217 previous research in professional rugby league that reported perceived fatigue, general

218 wellbeing and soreness to return to pre-game values within four days post-match. ${ }^{21}$ Other research in professional AF also reported perceived fatigue, stiffness, sleep quality, stress and general wellbeing to improve as gameday approached (i.e. as hours following the previous match increased). ${ }^{8}$ Another notable finding of the current study was that perceived sleep quality, motivation and fatigue displayed relatively low SNRs at all time points (SNR: 1.3 to 
motivation and fatigue with relative caution. Perceived sleep quality, fatigue and motivation also displayed the highest typical error of the five elements examined in this study, indicating poor reliability. Collectively, our findings suggest that perceived stress and soreness provide the most useful information regarding a player's perceived readiness to train on the basis of acceptable reliability and relatively good responsiveness to training stressors.

Submaximal heart rate tests are considered valid measurements of aerobic fitness in individual and team sport athletes. ${ }^{28,29}$ We found the typical test error in HRex and HRR to be considerably higher than those reported in previous research using similar protocols, ${ }^{17}$ with disparities possibly due to subtle differences in test protocols, the smaller sample of players and the different manufacturer of the heart rate monitors used in the present study. Moreover, the previous study performed testing on an artificial turf surface indoors in contrast to our testing being conducted outdoors, the latter being less of a controlled testing environment.

238 Additionally, different temperatures on testing days in the present study (20.0 degrees Celsius and 25.5 degrees Celsius, respectively) may further explain the difference in findings. Nonetheless, despite the relatively high typical error reported in our study, HRex and HRR displayed acceptable SNRs, indicating that the test can identify changes that exceed the typical error. Notably, HRex displayed greater sensitivity than HRR (5.3 compared to 1.4), therefore we suggest using heart rate during submaximal exercise in preference to heart rate during recovery as a training monitoring test in professional AF. Indeed, our study demonstrates that this is a non-invasive test ${ }^{30}$ and hence we recommend the inclusion of a submaximal heart rate recovery test in monitoring systems of professional AF players. 
248 The eccentric hamstring strength test in the present study demonstrated lower typical error 249 (CV\%: $2.9-4.2 \%)$ compared to previous research in recreational athletes $(\mathrm{CV} \%: 5.8-8.5)^{16}$ 250 and professional footballers (CV\%: $4.3-6.3) .{ }^{9}$ In contrast with the previous study, we assessed 251 reliability using highly-trained athletes who were very familiar with testing protocols. Notably, 252 left limb force production displayed a poorer reliability (CV\%: 4.2) and subsequently a lower 253 SNR than right limb (CV\%: 3.3) and average force (CV\%: 2.9) respectively, which may be due to the specific bilateral strength imbalances of the observation group. Our finding agrees with a previous study in professional footballers that reported a lower test error for force values collected from players' dominant leg (CV\%: 4.3) compared to non-dominant leg (CV\%: 5.4). This supports monitoring of individual changes in dominant and non-dominant leg hamstring strength in professional football. Collectively, our findings suggest that the test used in the present study is a reliable and sensitive method to assess eccentric hamstring strength in professional AF players throughout a competition season.

Previous research has assessed the reliability of the $\mathrm{CMJ}$ test ${ }^{20}$ and relationships between CMJ performance and external load ${ }^{18}$ in team sport athletes, no studies have determined the sensitivity of this test in professional AF players. The present study examined reliability and sensitivity of five CMJ variables, with concentric mean force (SNR: 2.3) and relative peak force (SNR: 2.5) displaying the greatest capability to detect changes that exceed the typical test error. Interestingly, these two variables also displayed similarly low typical test error (CV\%: 2.1 and 2.6, respectively) to those reported previously, ${ }^{20}$ suggesting they may be the most responsive $\mathrm{CMJ}$ measures for coaches and scientists of professional AF teams to monitor.

270 However, previous research in collegiate athletes reported lower inter day CVs than those reported in our study, with test $\mathrm{CV} \%$ ranging from $2.7-4.3 \%$ in relative peak power, relative peak force and relative mean force. ${ }^{23}$ The differences in findings may be explained by the 
273 design of the present study in measuring CMJ performance within a professional AF training environment in contrast to reliability research conducted in a laboratory setting on three occasions during a seven-day period used in previous research. ${ }^{23}$ We also examined the reliability and sensitivity of reactive strength index (RSI), which has been suggested as a superior measure to jump height and other force and power variables in assessing the stretch-

278 shortening cycle of athletes and therefore their explosiveness when jumping. ${ }^{31,32}$ Research in professional rugby league reported players with a greater RSI demonstrated superior force, power and impulse during both the concentric and eccentric phases of a $\mathrm{CMJ}$ in comparison to their lower RSI counterparts. ${ }^{32} \mathrm{We}$ found RSI to have relatively low typical error (CV\%: 7.0\%) and high sensitivity (SNR: 1.9), indicating it to be a useful global measure of CMJ performance in professional AF players.

While the results of this study provide information on the reliability and sensitivity of common measures for monitoring professional AF players, caution should be taken when generalising these findings. The current study did not relate these monitoring data against outcome measures (i.e. injury or performance), therefore further work is required to establish their efficacy as monitoring tools. Additionally, while the wellness measures examined in this study are commonly used and based on previous research, they have not been developed using accepted psychometric validation approaches, therefore it is recommended that changes exceeding the typical error in the measures reported in this study be interpreted alongside other monitoring data. Further, a possible confounding factor on our results was the collection of test-retest data obtained for eccentric hamstring strength and countermovement jump performance following a field training session. While conducting these tests on a non-training day is preferable, our methods reflect a typical training environment in professional AF football where eccentric 
297 testing is not conducted prior to main skills training sessions, providing strong ecological 298 validity for our results.

\section{Conclusion}

301 The present study examined the reliability and sensitivity of commonly used monitoring tools

302 in professional AF. Our findings provide a framework for assessing sensitivity of monitoring

303 tests and can inform practitioners on meaningful changes in results of these tests. While we 304 classified tests with a SNR of $1.0-1.5$ as acceptable, those that display a SNR of $>1.5$ will 305 provide practitioners with more useful information when assessing changes in constructs of 306 fitness and fatigue.

\section{Practical applications}

309 - Perceived wellness questionnaires, eccentric hamstring strength tests,

310 countermovement jump tests and submaximal heart rate recovery tests demonstrate $311 \quad$ acceptable to good sensitivity.

312 - Monitoring perceived sleep quality, motivation and fatigue via wellness questionnaires

313 provides little insight into the fitness and fatigue status of professional AF players.

314 - SNR analysis is a novel method of assessing the capacity of a measure to detect changes 315 that exceed typical test error when monitoring professional AF players. 


\section{References}

1. Taylor K, Chapman D, Cronin J, Newton M, Gill N. Fatigue monitoring in high performance sport: a survey of current trends. J Aust Strength Cond. 2012;20(1):1223.

2. Thorpe RT, Atkinson G, Drust B, Gregson W. Monitoring fatigue status in elite teamsport athletes: Implications for practice. Int J Sports Physiol Perform. 2017;12(Suppl 2):S227-s234.

3. Coutts A, Cormack S. Monitoring the training response. High-Performance Training for Sports. 2014:71-84.

4. Coutts AJ, Crowcroft, S., \& Kempton, T. Developing athlete monitoring systems: Theoretical basis and practical applications. Sport, Recovery, and Performance: Interdisciplinary Insights: Abingdon: Routledge; 2018.

5. Buchheit M, Voss SC, Nybo L, Mohr M, Racinais S. Physiological and performance adaptations to an in-season soccer camp in the heat: associations with heart rate and heart rate variability. Scand J Med Sport Sci. 2011;21(6):e477-485.

6. Edwards T, Spiteri T, Piggott B, Bonhotal J, Haff GG, Joyce C. Reliability and Sensitivity of Neuromuscular and Perceptual Fatigue Measures in Collegiate Men's Basketball. J Strength Cond Res. 2018. Publish Ahead of Print.

7. Lovell R, Siegler JC, Knox M, Brennan S, Marshall PW. Acute neuromuscular and performance responses to Nordic hamstring exercises completed before or after football training. J Sport Sci. 2016;34(24):2286-2294.

8. Gastin PB, Meyer D, Robinson D. Perceptions of wellness to monitor adaptive responses to training and competition in elite Australian football. J Strength Cond Res. 2013;27(9):2518-2526.

9. McCall A, Nedelec M, Carling C, Le Gall F, Berthoin S, Dupont G. Reliability and sensitivity of a simple isometric posterior lower limb muscle test in professional football players. J Sport Sci. 2015;33(12):1298-1304.

10. Currell K, Jeukendrup AE. Validity, reliability and sensitivity of measures of sporting performance. Sports Med. 2008;38(4):297-316.

11. Robertson S, Kremer P, Aisbett B, Tran J, Cerin E. Consensus on measurement properties and feasibility of performance tests for the exercise and sport sciences: a Delphi study. Sports Med. 2017;3(1):2-2.

12. Ryan S, Kempton T, Pacecca E, Coutts AJ. Measurement properties of an adductor strength assessment system in professional Australian footballers. Int J Sports Physiol Perform. 2018:1-13.

13. Hopkins WG. Measures of reliability in sports medicine and science. Sports Med. 2000;30(1):1-15.

14. Impellizzeri FM, Marcora SM. Test validation in sport physiology: lessons learned from clinimetrics. Int J Sports Physiol Perform. 2009;4(2):269-277.

15. Gallo TF, Cormack SJ, Gabbett TJ, Lorenzen CH. Self-reported wellness profiles of professional Australian football players during the competition phase of the season. $J$ Strength Cond Res. 2017;31(2):495-502.

16. Opar DA, Piatkowski T, Williams MD, Shield AJ. A novel device using the Nordic hamstring exercise to assess eccentric knee flexor strength: a reliability and retrospective injury study. J Orthop Phys Ther. 2013;43(9):636-640.

17. Veugelers KR, Naughton GA, Duncan CS, Burgess DJ, Graham SR. Validity and reliability of a submaximal intermittent running test in elite Australian football players. J Strength Cond Res. 2016;30(12):3347-3353. 
18. Cormack SJ, Mooney MG, Morgan W, McGuigan MR. Influence of neuromuscular fatigue on accelerometer load in elite Australian football players. Int J Sports Physiol Perform. 2013;8(4):373-378.

19. Cormack SJ, Newton RU, McGuigan MR. Neuromuscular and endocrine responses of elite players to an Australian Rules football match. Int J Sports Physiol Perform. 2008;3(3):359-374.

20. Cormack SJ, Newton RU, McGuigan MR, Doyle TL. Reliability of measures obtained during single and repeated countermovement jumps. Int J Sports Physiol Perform. 2008;3(2):131-144.

21. McLean BD, Coutts AJ, Kelly V, McGuigan MR, Cormack SJ. Neuromuscular, endocrine, and perceptual fatigue responses during different length between-match microcycles in professional rugby league players. Int J Sports Physiol Perform. 2010;5(3):367-383.

22. Crowcroft S, McCleave E, Slattery K, Coutts AJ. Assessing the Measurement Sensitivity and Diagnostic Characteristics of athlete-monitoring tools in national swimmers. Int J Sports Physiol Perform. 2017;12(Suppl 2):S295-s2100.

23. Gathercole R, Sporer B, Stellingwerff T, Sleivert G. Alternative countermovementjump analysis to quantify acute neuromuscular fatigue. Int $J$ Sports Physiol Perform. 2015;10(1):84-92.

24. Heishman AD, Daub BD, Miller RM, Freitas EDS, Frantz BA, Bemben MG. Countermovement jump reliability performed with and without an arm swing in NCAA Division 1 intercollegiate basketball players. J Strength Cond Res. 2018. Publish Ahead of Print.

25. Kempton T, Sirotic AC, Coutts AJ. An integrated analysis of match-related fatigue in professional rugby league. J Sport Sci. 2015;33(1):39-47.

26. Hopkins WG, Marshall SW, Batterham AM, Hanin J. Progressive statistics for studies in sports medicine and exercise science. Med Sci Sports Exerc. 2009;41(1):3-13.

27. Roe G, Darrall-Jones J, Till K, Phibbs P, Read D, Weakley J, Jones B. Between-days reliability and sensitivity of common fatigue measures in rugby players. Int J Sports Physiol Perform. 2016;11(5):581-586.

28. Cornforth DJ, Robinson DJ, Spence I, Jelinek HF. Heart rate recovery in decision support for high performance athlete training schedules. Int J Inf Know Mgmt. 2014;9:193-2017.

29. Buchheit M, Racinais S, Bilsborough JC, et al. Monitoring fitness, fatigue and running performance during a pre-season training camp in elite football players. $J \mathrm{Sci}$ Med Sport. 2013;16(6):550-555.

30. Buchheit M. Monitoring training status with HR measures: do all roads lead to Rome? Front Physiol. 2014;5:73-73.

31. Flanagan E, Comyns T. The use of contact time and the reactive strength index to optimize fast stretch-shortening cycle training. Strength Cond J. 2008;30(5):32-38.

32. McMahon JJ, Jones PA, Suchomel TJ, Lake J, Comfort P. Influence of the reactive strength index modified on force- and power-time curves. Int J Sports Physiol Perform. 2018;13(2):220-227. 
Table 1: Test-retest reliability of heart rate recovery, perceptual wellness, countermovement jump and eccentric hamstring strength tests.

\begin{tabular}{|c|c|c|c|c|c|}
\hline Monitoring measure & Mean & SD & Subjects & CV/TE $(90 \% \mathrm{CI})$ & ICC \\
\hline \multicolumn{6}{|l|}{ Heart Rate } \\
\hline HRex (bpm) & 88.8 & 5.3 & 16 & $1.2 \%(0.9,1.7)$ & 0.95 \\
\hline \multicolumn{6}{|l|}{ Perceptual Wellness } \\
\hline Perceived stress (z-score) & 0.0 & 0.1 & 14 & $0.07(0.06,0.11)$ & 0.45 \\
\hline Perceived soreness (z-score) & 0.2 & 0.5 & 14 & $0.29(0.22,0.43)$ & 0.77 \\
\hline Perceived sleep quality (z-score) & -0.1 & 1.1 & 14 & $0.71(0.54,1.05)$ & 0.64 \\
\hline Perceived fatigue (z-score) & 0.2 & 0.8 & 14 & $0.65(0.50,0.97)$ & 0.30 \\
\hline \multicolumn{6}{|l|}{ Countermovement Jump } \\
\hline Peak jump height $(\mathrm{cm})$ & 38.2 & 5.2 & 18 & $3.9 \%(3.1,5.5)$ & 0.93 \\
\hline Mean concentric force $(\mathrm{N})$ & 1792.9 & 195.4 & 18 & $2.1 \%(1.7,3.0)$ & 0.97 \\
\hline Left limb hamstring strength $(\mathrm{N})$ & 391.4 & 63.4 & 18 & $4.2 \%(3.3,5.9)$ & 0.89 \\
\hline Right limb hamstring strength (N) & 401.1 & 67.5 & 18 & $3.3 \%(2.6,4.7)$ & 0.87 \\
\hline Average limb hamstring strength $(\mathrm{N})$ & 396.4 & 63.9 & 18 & $2.9 \%(2.2,4.0)$ & 0.92 \\
\hline
\end{tabular}

HRex: exercise heart rate; HRR: heart rate recovery; bpm: heart beats per minute; cm: centimeters; N: Newtons; W/kg: watts per kilogram of body weight; N/kg: Newtons per kilogram of body weight; $\mathrm{m} / \mathrm{s}$ : metres per second; SD: standard deviation; CV: coefficient variation percentage; CI: confidence interval; ICC: intraclass coefficient. 
Table 2: Weekly variation and signal-to-noise ratio of heart rate recovery, countermovement jump and eccentric hamstring strength tests.

\begin{tabular}{|c|c|c|c|c|c|c|}
\hline Monitoring measure & Mean & SD & Subjects & $\mathrm{CV}(90 \% \mathrm{CI})$ & SNR & SNR Rating \\
\hline \multicolumn{7}{|l|}{ Heart Rate $(\mathrm{n}=176)$} \\
\hline HRex (bpm) & 81.6 & 6.2 & 41 & $7.4(6.5,8.9)$ & 5.3 & Good \\
\hline HRR (bpm) & 35.4 & 10.6 & 41 & $23.9(16.2,28.6)$ & 1.4 & Acceptable \\
\hline \multicolumn{7}{|l|}{ Countermovement Jump $(\mathrm{n}=206)$} \\
\hline Peak jump height (cm) & 38.0 & 4.4 & 35 & $5.9(5.4,6.6)$ & 1.5 & Acceptable \\
\hline Mean concentric force $(\mathrm{N})$ & 1806.4 & 225.8 & 35 & $4.9(4.5,5.5)$ & 2.3 & Good \\
\hline Reactive strength index (m/s) & 0.50 & 0.10 & 35 & $13.5(12.2,15.2)$ & 1.9 & Good \\
\hline Relative peak power (W/kg) & 53.7 & 5.1 & 35 & $5.4(4.9,6.1)$ & 1.5 & Good \\
\hline Relative peak force (N/kg) & 25.3 & 2.5 & 35 & $6.4(5.8,7.2)$ & 2.5 & Good \\
\hline \multicolumn{7}{|l|}{ Eccentric Hamstring Strength $(\mathrm{n}=543)$} \\
\hline Left limb hamstring strength $(\mathrm{N})$ & 378.2 & 78.1 & 39 & $8.4(7.9,8.9)$ & 2.0 & Good \\
\hline Right limb hamstring strength (N) & 387.1 & 74.6 & 39 & $7.9(7.4,8.4)$ & 2.4 & Good \\
\hline Average limb hamstring strength $(\mathrm{N})$ & 382.6 & 74.0 & 39 & $7.0(6.6,7.4)$ & 2.4 & Good \\
\hline
\end{tabular}

SNR: signal-to-noise ratio; HRex: exercise heart rate; HRR: heart rate recovery; bpm: heart beats per minute; cm: centimeters; N: Newtons; W/kg: watts per kilogram of body weight; N/kg: Newtons per kilogram of body weight; m/s: metres per second; SD: standard deviation; CV: coefficient variation percentage; CI: confidence interval. 
Table 3: Weekly variation and signal-to-noise ratio of perceptual wellness measures at 48, 72 and 96 hours post-match.

\begin{tabular}{|c|c|c|c|c|c|c|}
\hline Monitoring measure & Mean & $\mathrm{SD}$ & Subjects & TE $(90 \% \mathrm{CI})$ & SNR & SNR Rating \\
\hline \multicolumn{7}{|l|}{48 hours post-match $(\mathrm{n}=576)$} \\
\hline Perceived stress (z-score) & 0.0 & 0.7 & 42 & $0.78(0.73,0.83)$ & 11.1 & Good \\
\hline Perceived soreness (z-score) & 0.0 & 0.9 & 42 & $0.94(0.88,1.03)$ & 3.2 & Good \\
\hline Perceived motivation (z-score) & 0.0 & 0.8 & 42 & $0.78(0.73,0.83)$ & 1.3 & Acceptable \\
\hline Perceived sleep quality (z-score) & 0.0 & 0.9 & 42 & $0.92(0.87,0.97)$ & 1.3 & Acceptable \\
\hline Perceived fatigue (z-score) & 0.0 & 0.9 & 42 & $0.91(0.87,0.97)$ & 1.4 & Acceptable \\
\hline \multicolumn{7}{|l|}{72 hours post-match $(\mathrm{n}=511)$} \\
\hline Perceived stress (z-score) & 0.0 & 0.7 & 42 & $0.72(0.68,0.78)$ & 10.2 & Good \\
\hline Perceived soreness (z-score) & 0.0 & 0.9 & 42 & $0.91(0.86,0.98)$ & 3.1 & Good \\
\hline Perceived motivation (z-score) & 0.0 & 0.9 & 42 & $0.85(0.80,0.91)$ & 1.4 & Acceptable \\
\hline Perceived sleep quality (z-score) & 0.0 & 0.9 & 42 & $0.92(0.87,0.98)$ & 1.3 & Acceptable \\
\hline Perceived fatigue (z-score) & 0.0 & 0.9 & 42 & $0.92(0.87,0.98)$ & 1.4 & Acceptable \\
\hline \multicolumn{7}{|l|}{96 hours post-match $(\mathrm{n}=431)$} \\
\hline Perceived stress (z-score) & 0.0 & 0.1 & 42 & $0.09(0.09,0.10)$ & 1.3 & Acceptable \\
\hline Perceived soreness (z-score) & 0.0 & 0.8 & 42 & $0.86(0.81,0.93)$ & 2.9 & Good \\
\hline Perceived motivation (z-score) & 0.0 & 0.7 & 42 & $0.76(0.71,0.93)$ & 1.3 & Acceptable \\
\hline Perceived sleep quality (z-score) & 0.0 & 0.9 & 42 & $0.89(0.83,0.95)$ & 1.3 & Acceptable \\
\hline Perceived fatigue (z-score) & 0.0 & 0.9 & 42 & $0.95(0.89,1.02)$ & 1.5 & Good \\
\hline
\end{tabular}

SNR: signal-to-noise ratio: SD: standard deviation; TE: typical error; CI: confidence interval. 